\title{
Absence of a large superconductivity-induced gap in magnetic fluctuations of $\mathrm{Sr}_{2} \mathrm{RuO}_{4}$
}

\author{
S. Kunkemöller, ${ }^{1}$ P. Steffens, ${ }^{2}$ P. Link,${ }^{3}$ Y. Sidis, ${ }^{4}$ Z. Q. Mao, ${ }^{5,6}$ Y. Maeno, ${ }^{5}$ and M. Braden ${ }^{1, *}$ \\ ${ }^{1}$ II. Physikalisches Institut, Universität zu Köln, Zülpicher Str. 77, D-50937 Köln, Germany \\ ${ }^{2}$ Institut Laue Langevin, 71 avenue des Martyrs, 38000 Grenoble, France \\ ${ }^{3}$ Heinz Maier-Leibnitz Zentrum, Technische Universität München, Lichtenbergstrasse 1, 85748 Garching, Germany \\ ${ }^{4}$ Laboratoire Léon Brillouin, C.E.A./C.N.R.S., F-91191 Gif-sur-Yvette CEDEX, France \\ ${ }^{5}$ Department of Physics, Graduate School of Science, Kyoto University, Kyoto 606-8502, Japan \\ ${ }^{6}$ Department of Physics, Tulane University, New Orleans, LA 70118, USA
}

\begin{abstract}
Inelastic neutron scattering experiments on $\mathrm{Sr}_{2} \mathrm{RuO}_{4}$ determine the spectral weight of the nesting induced magnetic fluctuations across the superconducting transition. There is no observable change at the superconducting transition down to an energy of $\sim 0.35 \mathrm{meV}$, which is well below the $2 \Delta$ values reported in several tunneling experiments. At this and higher energies magnetic fluctuations clearly persist in the superconducting state. Only at energies below $\sim 0.3 \mathrm{meV}$ evidence for partial suppression of spectral weight in the superconducting state can be observed. This strongly suggests that the one-dimensional bands with the associated nesting fluctuations do not form the active, highly gapped bands in the superconducting pairing in $\mathrm{Sr}_{2} \mathrm{RuO}_{4}$.
\end{abstract}

$\mathrm{Sr}_{2} \mathrm{RuO}_{4}$ is one of the best studied unconventional superconductors [1 5] but its pairing symmetry and mechanism still remain a subject of very active debate. There is newly added evidence in favor of the most advocated symmetry of the superconducting order, namely the spintriplet chiral p-wave symmetry, such as the increase in the Knight shift expected in the equal-spin-pairing (ESP) triplet state [6], observation of the surface density of states consistent with the chiral edge state [7], and the magnetization steps corresponding to the half-quantum fluxoids [8]. On the other hand, there are results challenging the p-wave pairing scenario, such as the strong limiting of the in-plane upper critical fields [9], the firstorder superconducting transition [10, 11], and the absence of the chiral edge current [12]. At present, there seems no symmetry model which can explain all the experimental facts available. If the most advocated symmetry of the superconducting order is correct, $\mathrm{Sr}_{2} \mathrm{RuO}_{4}$ would be a topological superconductor proposed as a promising candidate for quantum computing [13, 14].

Another prominent feature of $\mathrm{Sr}_{2} \mathrm{RuO}_{4}$ is that its normal state is quantitatively well characterized as a quasitwo-dimensional (Q2D) Fermi liquid [2, 3]. The Fermi surface consists of three cylindrical sheets [2]: two originate from the $d_{x z}$ and $d_{y z}$ orbitals, called the $\alpha$ and $\beta$ bands, and retain a quasi-one-dimensional (Q1D) character as well; the other one from the $d_{x y}$, called the $\gamma$ band, shows a Q2D character. All three bands disperse weakly along the interlayer $c$ direction [15]. In such a multiband system with distinct orbital symmetries, superconductivity may be strongly orbital dependent [16]. The strong nesting between the Q1D bands results in strongly enhanced spin-density wave (SDW) fluctuations [17-22] and even minor chemical substitution leads to static ordering of this SDW instability with the moment along the c direction. Only $2.5 \%$ of Ti induce this SDW phase 23, 24], and recent muSR experiments and neutron scattering studies show that the same magnetic order oc- curs upon replacing Sr with isovalent Ca [25, 26]. Such spin fluctuations originating from the nesting of the Q1D Fermi surface sheets cannot easily lead to the most likely chiral superconducting state [2]. The equal-spin p-wave pairing scenario is based on quasi-ferromagnetic correlations associated with the $\gamma$ band, and amongst the various p-wave possibilities a chiral (and topological) state, $k_{x}+i k_{y}$, was proposed to explain various experiments 2, 3]. Evidence for strong quasi-ferromagnetic fluctuations can be found in susceptibility [2, 3] and NMR measurements [27], but a thorough study of such fluctuations still lacks. Thus, one important step toward resolving the apparent controversy is to identify which of the bands are mainly responsible for the superconductivity.

Many attempts were made to reconcile the discrepancy between the pairing symmetry and the apparently dominant magnetic fluctuations [2, 3, 5]. Treating the on-site Coulomb repulsion within perturbation theory corroborates the scenario of $p$-wave pairing mainly arising in the Q2D band [28]. This scenario is challenged by Raghu et al., who apply renormalization group techniques and discuss orbital and charge fluctuations in the Q1D bands as the main ingredient 29]. These calculations were extended by Scaffidi et al. [30] to include interband and spin-orbit coupling yielding similar sized gaps on all bands without tuning of parameters. In contrast the recent analysis by Huo, Rice and Zhang argues in favor of superconductivity arising in the Q2D bands with the nesting fluctuations perturbing the superconductivity 31]. Experimentally, the observation of a strong enhancement of the superconducting $T_{c}$ (by a factor 2!) under both tensile and compressive strain [32] may suggest a dominant influence of the van Hove singularity in the Q2D bands associated with the ferromagnetic instability. The question which bands drive superconductivity in $\mathrm{Sr}_{2} \mathrm{RuO}_{4}$ remains as open and fascinating as ever [33].

Inelastic neutron scattering (INS) can yield valuable information concerning the role of the different bands in 

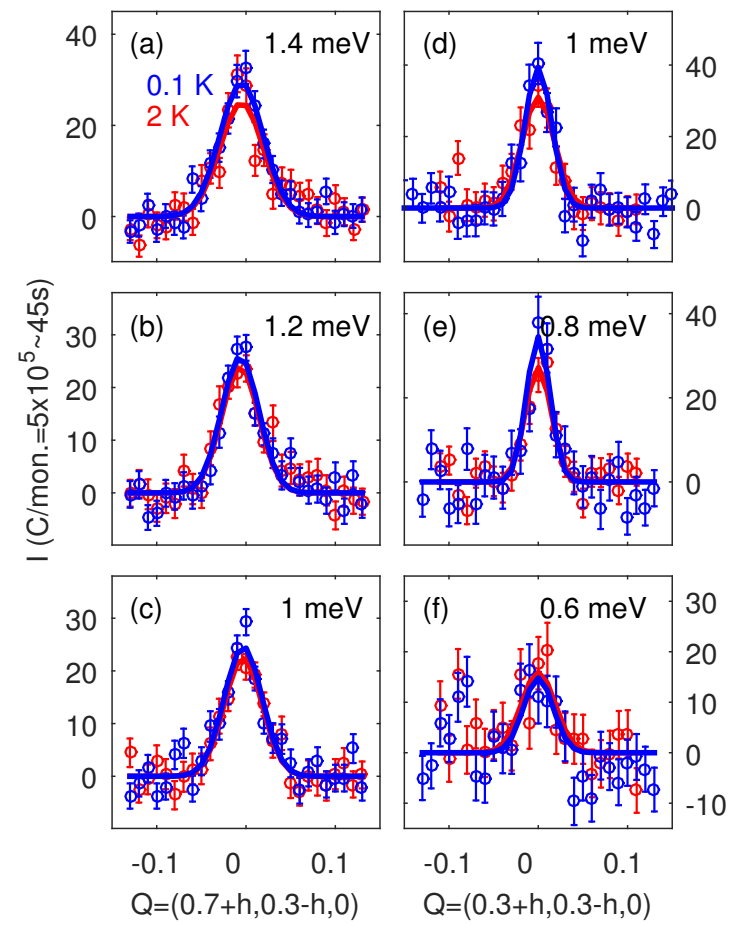

FIG. 1: Constant-energy scans obtained on THALES with $k_{f}=1.57 \AA^{-1}$ using the PG-PG configuration. Intensity profiles were fitted by the sum of a Gaussian peak and a curved background, which was assumed identical at both temperatures and subtracted from the data.

the pairing 31]. If superconductivity directly arises from the Q1D bands as active bands, which thus exhibit a large gap, there must be a clear impact on the associated incommensurate magnetic excitations. Several calculations explicitly predict the occurrence of a resonance mode in at least one of the spin excitation channels for $p$ wave superconducting symmetry [31, 37 39]. On the other hand, if superconductivity is mainly driven by the Q2D band associated with ferromagnetic fluctuations, a lower gap in the Q1D bands and only a small impact on the magnetic fluctuations is expected [16, 31]. Here we report INS experiments across the superconducting transition in $\mathrm{Sr}_{2} \mathrm{RuO}_{4}$, which clearly show that nestinginduced magnetic fluctuations only sense a very small gap suggesting that the Q1D bands are not the active ones in the superconducting pairing.

The difficulty of INS experiments on the magnetic response in the superconducting state of $\mathrm{Sr}_{2} \mathrm{RuO}_{4}$ consists in the weakness of the signal combined with the high resolution needed. The INS intensity is given by the imaginary part of the generalized susceptibility, $\chi^{\prime \prime}(\mathbf{Q}, E)$, multiplied by the Bose factor [19]:

$$
\frac{d^{2} \sigma}{d \Omega d E}=\frac{k_{f} r_{0}^{2} F^{2}(Q)}{k_{i} \pi\left(g \mu_{B}\right)^{2}} \frac{2 \cdot \chi^{\prime \prime}(\mathbf{Q}, E)}{1-\exp \left(-E / k_{b} T\right)},
$$

where we ignore the spin anisotropy of the magnetic susceptibility [21] ( $k_{i}$ and $k_{f}$ denote incoming and final neu-
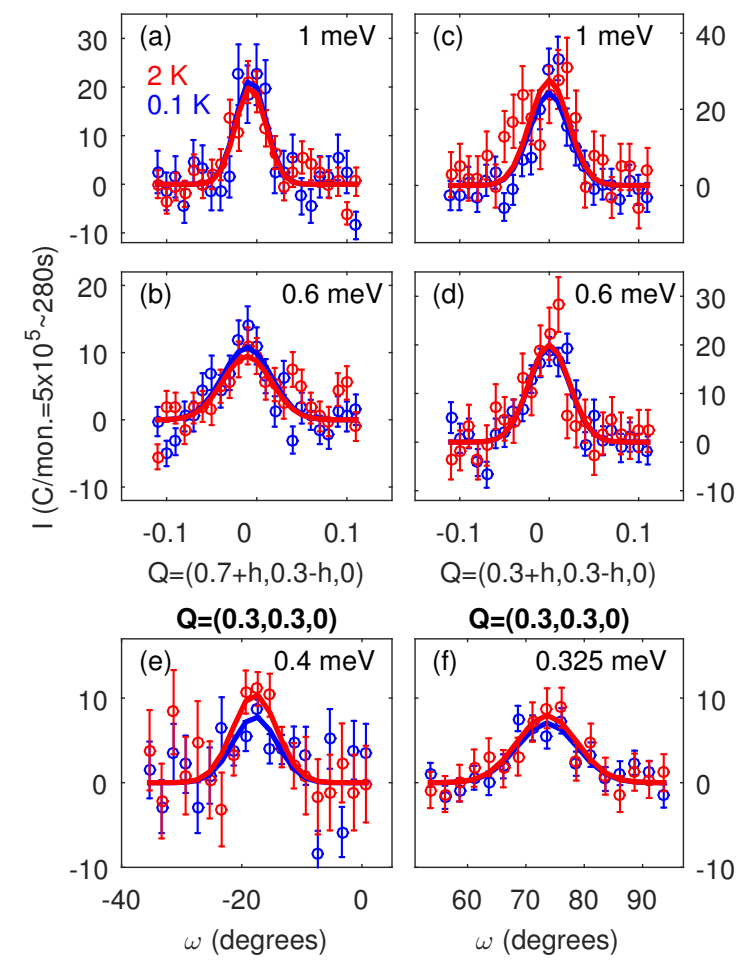

FIG. 2: (a-d) Constant-energy scans obtained on THALES with $k_{f}=1.57 \AA^{-1}$ using the Si-PG configuration. A flat background was subtracted from the data. (e-f) Inelastic rocking scans using the Si-PG configuration. The sample was rotated through the $(0.3,0.3,0) E=0.4$ and $0.325 \mathrm{meV}$ positions at the center of the scans yielding a flat background that was subtracted from the data.

tron momentum, $F(Q)$ the magnetic form factor of $\mathrm{Ru}$ at the scattering vector and $\left.r_{0}{ }^{2}=0.29 \cdot 10^{-28} \mathrm{~m}^{2}\right)$. The nesting-induced magnetic excitations at $\mathbf{q}_{\text {inc }}$ follow a single relaxor behavior [18, 19, 21, 22]:

$$
\chi^{\prime \prime}\left(\mathbf{q}_{i n c}, E\right)=\chi^{\prime}\left(\mathbf{q}_{i n c}, 0\right) \frac{\Gamma E}{\Gamma^{2}+E^{2}},
$$

which is maximum at the characteristic energy $\Gamma$ and almost linear for much lower energies. INS experiments in the normal state indicate strong magnetic scattering at the nesting vector, $\mathbf{q}_{i n c}$, with the characteristic energy decreasing towards low temperatures. But this softening stops at $\Gamma \sim 6 \mathrm{meV}$, which is well above the values of the superconducting gap [18, 19, 21]. Therefore, the INS signal in the range, where one may expect an impact of the superconducting gap, is very small. In addition, the experiment requires a high energy resolution in order to study this region close to the strong elastic response, which considerably reduces the INS intensity. Due to these difficulties the previous INS experiments on $\mathrm{Sr}_{2} \mathrm{RuO}_{4}$ in the superconducting phase yielded reliable statistics on the nesting fluctuations only for energy transfer above $\sim 1 \mathrm{meV}[19]$.

INS experiments were carried out on the PANDA 
triple-axis spectrometer at the Forschungsreaktor $\mathrm{Mu}$ nich II and at the recently upgraded THALES instrument at the Institut Laue Langevin. In all experiments we used an assembly of $12 \mathrm{Sr}_{2} \mathrm{RuO}_{4}$ crystals with a total volume of $2.2 \mathrm{~cm}^{3}$. The crystals were grown at Kyoto University using a floating-zone image furnace and similar crystals were studied in many different experiments [2, 3]. We choose the $[100] /[010]$ scattering geometry, because this yields the best INS signal due to the integration along the vertical direction along $c$ where little modulation of magnetic response is expected. For all experiments the crystal assembly was cooled with a dilution refrigerator attaining minimum temperature of the order of $\sim 50 \mathrm{mK}$. There is some impact on the neutron absorption on the sample temperature of the order of $10 \mathrm{mK}$, which, however, is negligible compared to the transition temperature. On PANDA we mostly used a final momentum of $k_{f}=1.2 \AA^{-1}$ to obtain sufficient resolution and pyrolitic graphite (PG) (002) as monochromator and analyzer. In order to decrease the background a $\mathrm{BeO}$ filter was put in front of the analyzer and a Be filter between monochromator and sample. On THALES a much better intensity to background ratio was achieved, but some residual background at low energies remained when using PG (002) monochromator and analyzer crystals (PG-PG configuration) even for rather small values of the final momentum. In order to further suppress this low-energy background we included a radial collimator and a Be filter in front of the analyzer and we used a $\mathrm{Si}$ (111) monochromator (SI-PG configuration). We applied vertical and horizontal focusing at both the monochromator and analyzer. In addition, a velocity selector in front of the monochromator was inserted to suppress higher order contaminations. Most scans on THALES were performed with a fixed final momentum of $k_{f}=1.57 \AA^{-1}$ where the Be filter effectively cuts all neutrons with only slightly larger final energy. Some scans were performed by scattering at the sample and at the analyzer in the same sense (U configuration), which reduces the background as the detector is positioned farther away from the direct beam, but slightly worsens the resolution.

In spite of serious efforts the measurements on PANDA considerably suffered from the background scattering. Scans at the scattering vectors of $(0.3,0.3,0)$ and $(0.7,0.7,0.4)$ did not yield any indication for a superconductivity induced change at $T_{c}$ above $\mathrm{E} \sim 0.6 \mathrm{meV}$ but the achieved statistics at lower energy remained insufficient to characterize the weak magnetic signal. In the following we will therefore focus on the results obtained on THALES which exhibit significantly better statistics.

Figures 1 and 2 show constant energy scans for temperatures above and below the superconducting transition. The data in Fig. 1 were taken with the PG-PG configuration on THALES (energy resolution at the elastic line $\Delta E_{0}=0.20 \mathrm{meV}$ full width at half maximum) and those in Fig. 2 with the SI-PG configuration, which yields a lower background at small energies and improves the resolution $\left(\Delta E_{0}=0.12 \mathrm{meV}\right)$ but considerably reduces the signal. With the dilution refrigerator cryostat used in these experiments, it is not possible to obtain sufficient temperature stability in the range 1.2 to $1.6 \mathrm{~K}$, therefore we could not follow the signals close to $T_{c}$. The data shown in Fig. 1 and Fig. 2 unambiguously show that the nesting related fluctuations in the energy range 0.6 to $1 \mathrm{meV}$ can be easily studied by our INS experiment and that this signal is not affected by the superconducting transition concerning neither the intensity nor the width. We have studied the nesting signal at the two scattering vectors $\mathbf{Q}=(0.3,0.3,0)$ and $(0.7,0.3,0)$ which are not equivalent due to the centering of the body centered lattice in $\mathrm{Sr}_{2} \mathrm{RuO}_{4}$ and due to the lower form factor at the latter reducing the magnetic signal. Because of the quasi-twodimensional nature of the magnetic correlations in $\mathrm{Sr}_{2} \mathrm{RuO}_{4}$, however, one does not expect an essential difference, and the signal at both scattering vectors is comparable and in particular there is no change at the superconducting transition, $T_{c}=1.4 \mathrm{~K}$ for energies above $0.6 \mathrm{meV}$ at both $\mathbf{Q}$ values.

Experiments at lower energy transfer are more difficult as described above. Since the background depends on the length of the scattering vector (i.e. the scattering angle), it is not constant in a straight transversal constantenergy scan like those shown in Fig. 1 and 2(a-d) but may peak at the scan center. Therefore, we performed inelastic rocking scans by turning the sample with fixed $|\mathbf{Q}|$, see Fig. 2(e-f). These scans posses a flat background and clearly confirm that magnetic scattering persists in the superconducting state essentially unchanged down to energies of the order of $0.325 \mathrm{meV}$. Note that the Bose factor explains a small intensity reduction between 2 and $0.1 \mathrm{~K}$ of 1.18 and 1.11 at $E=0.325$ and $0.4 \mathrm{meV}$ respectively, so that the data do not yield any significant reduction of spectral weight even at $0.325 \mathrm{meV}$.

Figure 3 resumes the energy dependence of the magnetic nesting signal. Figure 3(a) shows the fitted peak heights of the constant- $E$ scans taken in different configurations at the two scattering vectors. In order to compare data taken at different $\mathbf{Q}$ positions, in different configurations (scattering sense at the analyser) and in different runs, intensities are normalized to the values at $1 \mathrm{meV}$ and $2 \mathrm{~K}$. The peak heights at larger energies remain unchanged upon entering the superconducting state while evidence for partial suppression of spectral weight is observed below $\sim 0.3 \mathrm{meV}$. Figure $3(\mathrm{~b})$ shows constant Q-scans taken at the nesting scattering vector $(0.3,0.3,0)$ above and below the superconducting transition as well as a background scan taken at a scattering vector of the same length but rotated 16 degrees away from the nesting position. Subtracting this background signal from that obtained at the nesting $\mathbf{Q}$-position we can deduce the magnetic signal at both temperatures, see Fig. 3(c). This analysis shows that the nesting scattering remains 

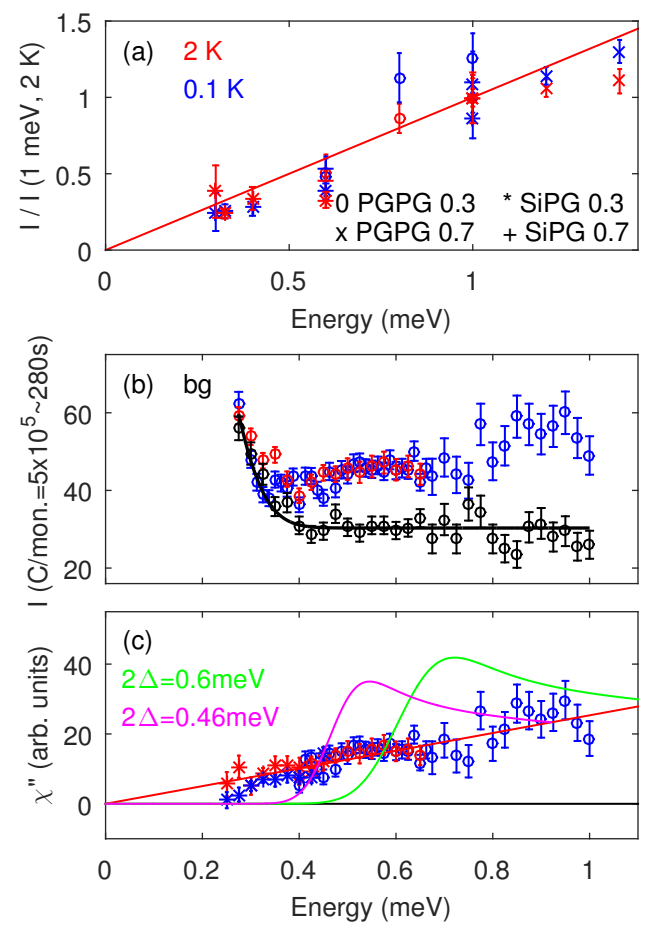

FIG. 3: (a) Fitted Gaussian peak heights obtained from the constant- $E$ scans taken with the two configurations at the two scattering vectors. In order to allow for comparison, the data were normalized to the values at $1 \mathrm{meV}$ and $2 \mathrm{~K}$ and a correction for the Bose factor was applied. (b) Constant-Q scans obtained with $k_{f}=1.57 \AA^{-1}$ using the Si-PG configuration. Blue and red symbols denote the data taken at $\mathbf{Q}=(0.3,0.3,0)$ above and below the superconducting transition, respectively, and black symbols denote background intensity observed at a $\mathbf{Q}$ vector of the same length but rotated by $16^{\circ}$ with respect to the $\mathbf{Q}$ position of the nesting response. (c) Magnetic signal at $\mathbf{Q}=(0.3,0.3,0)$ obtained by subtracting the background signal and by correcting for the Bose factor. Straight lines in (a) and (c) denote the linear relation $\chi^{\prime \prime} \propto E$ expected for the single relaxor at low energy, see equation (2). The magenta and green lines in (c) correspond to the calculated magnetic response in the case of active Q1D bands [31], which results in a resonance excitation at the energy of twice the superconducting gap taken at the weak coupling BCS value $0.46 \mathrm{meV}$ and at the value observed in tunneling experiments $(0.6 \mathrm{meV}$, see text). The theoretical result was folded with the experimental resolution. Data in (b) and part of the data in (c) (circles) were taken in U-configuration yielding lower background at energies above $0.4 \mathrm{meV}$ and slightly reduced energy resolution, $\Delta E_{0}=0.16 \mathrm{meV}$, while the lowenergy part of (c) was recorded in z-configuration (stars) in a dedicated experiment. In this $\mathrm{z}$-configuration the background remains flat and a better statistics could be reached resulting in much smaller error bars.

essentially unchanged for energies above $\sim 0.325 \mathrm{meV}$. The constant Q-scans data yield week evidence for partial suppression of spectral weight due to the opening of the superconducting gap only at very low energies, see Fig. 3(c), but additional studies are desirable.
The magnetic response of an itinerant system corresponds to a particle-hole excitation, which in a superconductor must cross twice the superconducting gap, $2 \Delta$. There have been several reports on the superconducting gap in $\mathrm{Sr}_{2} \mathrm{RuO}_{4}$ [7, 17, 34 36]: The first tunneling experiments were interpreted as evidence for very large gap and $\frac{2 \Delta}{k_{B} T_{c}}$ values [34, 35] while more recent studies conclusively suggest smaller values: Suderov et al. $2 \Delta=0.56 \mathrm{meV}$ [36], Kashiwara et al. $2 \Delta=0.93 \mathrm{meV}$ 7] and Firmo et al. $2 \Delta=0.7 \mathrm{meV}$ slightly above the weak coupling BCS value $2 \Delta=0.46 \mathrm{meV}$. None of the tunneling studies can safely identify the band carrying the largest gap, leaving the discussion about active and passive bands open. On the theoretical side, different studies arrive at nearly the same conclusion that opening the $p$-wave gap in the Q1D sheets results in a full suppression of spectral weight below $2 \Delta_{1 d}$ and even a resonance enhancement at or close to this value [12, 3739]. In Fig. 3(c) we include the calculation for a superconducting gap opening in the Q1D bands of 0.46 and $0.6 \mathrm{meV}$ [31] folded with the experimental resolution. Our results clearly contradict such picture. A resonance enhancement of the magnetic response in the superconducting state has been reported in several unconventional superconductors [40]. In particular in superconductors, in which the pairing appears mediated by well defined magnetic fluctuations such as the cuprates or the FeAsbased compounds, strong resonance modes are found [40]. Such a behavior can be excluded for the nesting scattering in $\mathrm{Sr}_{2} \mathrm{RuO}_{4}$ which exhibits no significant suppression of magnetic weight at energies well below the maximum $2 \Delta$ reported in the tunneling experiments or the weak coupling BCS value. It seems therefore very unlikely that the Q1D bands are the active ones for the superconducting pairing in $\mathrm{Sr}_{2} \mathrm{RuO}_{4}$. Instead the ferromagnetic fluctuations arising from the large density of states in the Q2D bands can imply superconductivity primordially in the Q2D bands. This scenario is supported by the fieldorientation dependence of the specific heat [41, 42] and NMR data 2, 3, 5, 33], and direct evidence for ferromagnetic fluctuations can be obtained from magnetization [2, 3] and polarized INS studies [43].

Nodes of the gap function may lead to persisting magnetic scattering in the superconducting state for energies below the maximum values of $2 \Delta$. But in the scenario of Q1D bands being the active ones for the superconducting pairing mediated by nesting induced fluctuations, some effect of the gap opening must be observed. The fact that there is no change in the magnetic scattering (below $20 \%$ for $E>0.325 \mathrm{meV}$ ) well below the observed maximum values of $2 \Delta, 7,17,34,36$ ] renders such a scenario very unlikely.

In conclusion we have studied the low-energy magnetic fluctuations associated with the nesting of Q1D bands in $\mathrm{Sr}_{2} \mathrm{RuO}_{4}$. The fact that we do not observe a significant change in this signal when passing the supercon- 
ducting transition disagrees with a scenario of nesting related fluctuations driving superconductivity primordially in the Q1D bands.

This work was supported by the Deutsche Forschungsgemeinschaft through CRC 1238 Project No. B04 and by the JSPS KAKENHI No. JP15H05852.

* e-mail: braden@ph2.uni-koeln.de

[1] Y. Maeno, H. Hashimoto, K. Yoshida, S. Nishizaki, T. Fujita, J. G. Bednorz and F. Lichtenberg, Nature (London) 372, 532 (1994).

[2] A. P. Mackenzie and Y. Maeno, Rev. Mod. Phys. 75, 657 (2003).

[3] Y. Maeno, S. Kittaka, T. Nomura, S. Yonezawa, and K. Ishida, J. Phys. Soc. Jpn. 81, 011009 (2012).

[4] Y. Liu and Z.-Q. Mao, Physica (Amsterdam) 514C, 339 (2015).

[5] C. Kallin and A. Berlinsky, Rep. Prog. Phys. 79, 054502 (2016).

[6] K. Ishida, M. Manago, T. Yamanaka, H. Fukazawa, Z. Q. Mao, Y. Maeno, and K. Miyake, Phys. Rev. B 92, 100502(R) (2015).

[7] S. Kashiwaya, H. Kashiwaya, H. Kambara, T. Furuta, H. Yaguchi, Y. Tanaka, and Y. Maeno, Phys. Rev. Lett. 107, 077003 (2011).

[8] J. Jang, D. G. Ferguson, V. Vakaryuk, R. Budakin, S. B. Chung, P. M. Golbart, and Y. Maeno, Science 331, 186 (2011).

[9] C. Rastovski, C. D. Dewhurst, W. J. Gannon, D. C. Peets, H. Takatsu, Y. Maeno, M. Ichioka, K. Machida, and M. R. Eskildsen, Phys. Rev. Lett. 111, 087003 (2013).

[10] S. Yonezawa, T. Kajikawa, and Y. Maeno, Phys. Rev. Lett. 110, 077003 (2013).

[11] S. Kittaka, A. Kasahara, T. Sakakibara, D. Shibata, S. Yonezawa, Y. Maeno, K. Tenya, and K. Machida, Phys. Rev. B 90, 220502(R) (2014).

[12] C. W. Hicks, J. R. Kirtley, T. M. Lippman, N. C. Koshnick, M. E. Huber, Y. Maeno, W. M. Yuhasz, M. B. Maple, and K. A. Moler, Phys. Rev. B 81, 214501 (2010).

[13] D. A. Ivanov, Phys. Rev. Lett. 86, 268 (2001).

[14] C. Nayak, S. H. Simon, A. Stern, M. Freedman, and S. D. Sarma, Rev. Mod. Phys. 80, 1083 (2008).

[15] C. N. Veenstra, Z.-H. Zhu, M. Raichle, B. M. Ludbrook, A. Nicolaou, B. Slomski, G. Landolt, S. Kittaka, Y. Maeno, J. H. Dil, I. S. Elfimov, M. W. Haverkort, and A. Damascelli, Phys. Rev. Lett. 112, 127002 (2014).

[16] D.F. Agterberg, T.M. Rice, and M. Sigrist, Phys. Rev. Lett. 78, 3374 (1997).

[17] I. A. Firmo, S. Lederer, C. Lupien, A. P. Mackenzie, J. C. Davis, and S. A. Kivelson, Phys. Rev. B 88, 134521 (2013).

[18] Y. Sidis, M. Braden, P. Bourges, B. Hennion, S. NishiZaki, Y. Maeno, and Y. Mori, Phys. Rev. Lett. 83, 3320 (1999).

[19] M. Braden, Y. Sidis, P. Bourges, P. Pfeuty, J. Kulda, Z.
Mao,and Y. Maeno, Phys. Rev. B 66, 064522 (2002).

[20] F. Servant, B. Fak, S. Raymond, J. P. Brison, P. Lejay, and J. Flouquet, Phys. Rev. B 65 , 184511 (2002).

[21] M. Braden, P. Steffens, Y. Sidis, J. Kulda, P. Bourges, S. Hayden, N. Kikugawa, and Y. Maeno, Phys. Rev. Lett. 92, 097402 (2004).

[22] K. Iida, M. Kofu, N. Katayama, J. Lee, R. Kajimoto, Y. Inamura, M. Nakamura, M. Arai, Y. Yoshida, M. Fujita, K. Yamada, and S.-H. Lee Phys. Rev. B 84, 060402(R) (2011).

[23] M. Minakata and Y. Maeno, Phys. Rev. B 63, 180504(R) (2001).

[24] M. Braden, O. Friedt, Y. Sidis, P. Bourges, M. Minakata and Y. Maeno, Phys. Rev. Lett. 88, 197002 (2002).

[25] J.P. Carlo, T. Goko, I. M. Gat-Malureanu, P. L. Russo, A. T. Savici, A. A. Aczel, G. J. MacDougall, J. A. Rodriguez, T. J. Williams, G. M. Luke, C. R. Wiebe, Y. Yoshida, S. Nakatsuji, Y. Maeno, T. Taniguchi and Y. J. Uemura, Nat. Mater. 11, 323 (2012).

[26] S. Kunkemöller, A. A. Nugroho, Y. Sidis and M. Braden, Phys. Rev. B 89, 045119 (2014)

[27] K. Ishida, H. Mukuda, Y. Minami, Y. Kitaoka, Z. Q. Mao, H. Fukazawa, and Y. Maeno, Phys. Rev. B 64, 100501 (2001).

[28] Y. Yanase, T. Jujo, T. Nomura, H. Ikeda, T. Hotta, and K. Yamada, Phys. Rep. 387, 1 (2003).

[29] S. Raghu, A. Kapitulnik, and S. A. Kivelson, Phys. Rev. Lett. 105, 136401 (2010).

[30] T. Scaffidi, J. C. Romers, and S. H. Simon, Phys. Rev. B 89, 220510(R) (2014)

[31] J.W. Huo, T.M. Rice, and F.-C. Zhang, Phys. Rev. Lett. 110, 167003 (2013).

[32] C. W. Hicks, D. O. Brodsky, E. A. Yelland, A. S. Gibbs, J. A. N. Bruin, M. E. Barber, S. D. Edkins, K. N. Nishimura, S. Yonezawa, Y. Maeno, and A. P. Mackenzie, Science 344, 283 (2014).

[33] C. Kallin, Rep. Prog. Phys. 75 (2012).

[34] F. Laube, G. Goll, H. v. Löhneysen, M. Fogelström, and F. Lichtenberg, Phys. Rev. Lett. 84, 1595 (2000).

[35] M. D. Upward, L. P. Kouwenhoven, A. F. Morpurgo, N. Kikugawa, Z. Q. Mao, and Y. Maeno, Phys. Rev. B 65, 220512(R) (2002).

[36] H. Suderow, V. Crespo, I. Guillamon, S. Vieira, F. Servant, P. Lejay, J. P. Brison, and J. Flouquet, New J. Phys. 11, 093004 (2009).

[37] Hae-Young Kee, J. Phys, Condens. Matter 12, 2279 (2000).

[38] D. K. Morr, P. F. Trautman, and M. J. Graf, Phys. Rev. Lett. 86, 5978 (2001).

[39] M. Yakiyama, and Y. Hasegawa, Phys. Rev. B 67, 014512 (2003).

[40] D. J. Scalapino, Rev. Mod. Phys. 84, 1383 (2012).

[41] K. Deguchi, Z. Q. Mao, and Y. Maeno, Phys. Rev. Lett. 92, 047002 (2004).

[42] K. Deguchi, Z. Q. Mao, H. Yaguchi, and Y. Maeno, Phys. Soc. Jpn. 73, 1313 (2004).

[43] M. Braden, P. Steffens, Y. Sidis, J. Kulda, P. Bourges, S. Hayden, N. Kikugawa, and Y. Maeno Phys. Rev. Lett. 92, 097402 (2004). 